\title{
Asymptotic Analysis of Outage Probability in Cognitive Radio Networks
}

\author{
Yaobin Wen, Sergey Loyka and Abbas Yongacoglu ${ }^{\dagger}$
}

\begin{abstract}
The aggregate interference distribution in cognitive radio networks is studied in a rigorous analytical way using the popular Poisson point process model. While a number of results are available for this model of regular (non-cognitive) networks, cognitive ones present an extra level of difficulty for the analysis, mainly due to the exclusion region around the primary receiver, which are typically addressed via various adhoc approximations (e.g. based on the interference cumulants) or via the large-deviation analysis. Unlike the previous studies, here we do not use ad-hoc approximations but rather obtain the asymptotic interference distribution in a systematic and rigorous way. This is in contrast to the large deviation analysis, which provides only the (exponential) order of scaling but not the outage probability itself. Unlike the cumulant-based analysis, our approach provides a guaranteed level of accuracy at the distribution tail. Additionally, our analysis also provides a number of novel insights. In particular, we demonstrate that there is a critical transition point below which the outage probability decays only polynomially but above which it decays exponentially. This provides a solid analytical foundation to the earlier empirical observations in the literature and also reveals how typical outage events occur in different regimes. In addition, the proposed asymptotic expressions are also shown to be accurate in the nonasymptotic regimes.
\end{abstract}

\section{INTRODUCTION}

As higher data rate services are required in wireless communications over a limited spectrum, there is a need for higher spectrum efficiency. Cognitive Radio (CR), which suggests allowing secondary users (SU) to share the spectrum currently not in use by a primary user (PU), is seen as the main way to overcome the overcrowded spectrum problem and to use it more efficiently [1]. Due to the uncertainty in the number of SUs and their locations, the PU performance may be seriously affected by the aggregate interference induced by the SUs. Therefore, its accurate modeling is important to design cognitive radio networks and also to estimate their potential benefits.

There is an extensive literature dealing with aggregate interference modeling of conventional (non-cognitive) networks [2]. The most popular elements of those models are a Poisson point process (to model the interferers' spatial locations) and the standard propagation path loss models. Based on that, Sousa and Silvester [3] studied the aggregate interference power obtaining its characteristic function $(\mathrm{CF})$ and concluding that it is an $\alpha$-stable random variable. Except for some special cases, the closed-form probability density function (PDF) is not available. Using the multivariate Lepage

$\dagger$ Y. Wen, S. Loyka and A. Yongacoglu are with the School of Electrical Engineering and Computer Science, University of Ottawa, Ontario, Canada, e-mails: ywen@rim.com, sergey.loyka@ieee.org, yongacog@site.uottawa.ca. series representation, Ilow and Hatzinakos [4] obtained the $\mathrm{CF}$ of the aggregate interference including the log-normal and Rayleigh fading effects and concluding that the aggregate interference is a spherically symmetric $\alpha$-stable random vector. Unlike the previous studies mainly based on the CF approach, Mordachev and Loyka [5] studied the tail of the aggregate interference distribution directly and found that, at the low outage regime, the aggregate interference is dominated by the nearest one, also under different fading models and interference cancelation mechanisms. Based on the direct asymptotic analysis, compact and accurate closed-form expressions for the outage probability were obtained and a number of insights were pointed out. The impact of optimum combining using the standard array processing techniques (e.g. MMSE spatial filter) has also been studied [6]. While in some special cases the aggregate interference distribution of a large wireless network approaches the Gaussian one [7], it is far from being accurate in general.

The studies above are limited to the conventional (non$\mathrm{CR}$ ) networks. The cognitive ones present an additional level of difficulties for the analysis due to the presence of the exclusion region around the PU receiver where no SUs are allowed to transmit (to ensure the PU's proper quality-ofservice), so that the distribution of the aggregate interference is not $\alpha$-stable any more and the models/results above cannot be applied directly. Those difficulties are mainly addressed in two typical ways: via various ad-hoc approximations (e.g. using the interference distribution cumulants found from its CF) [8][10] or via the large deviation analysis [11]. Additionally, numerical simulations provide a wealth of empirical observations [9]. Since a direct analysis of the interference distribution is challenging while the characteristic function is much more amenable to the analysis, from which its cumulants can be found, this approach was adopted in [8] and [10] to obtain approximations of the interference distribution based on the Edgeworth expansion and truncated stable distributions respectively. While this approach predicts the main body of the distribution well, its accuracy deteriorates significantly at the distribution tail, i.e. at the practically-important low-outage regime (high quality-of-service for the PU). This happens because a limited number of cumulants (typically two) cannot represent the tail sufficiently well. On the other hand, the use of the large deviation analysis allows one to predict the (exponential) rate of decay of the distribution tail [11] but not the distribution itself (as all constants and slowly-varying functions are neglected in the analysis).

To overcome these difficulties, we develop a direct approach 
to the outage probability analysis based on the heavy tail and saddle-point approximation theories. The advantage of this approach is that the distribution tail can be found in an explicit closed-form with a guaranteed level of accuracy, to the best of our knowledge, for the first time. Additionally, this analysis provides a number of insights and the expressions are also accurate in the non-asymptotic regime. Important geometrical and system parameters affecting critically the outage probability are clearly identified.

A similar CR scenario has been studied in [13][14] based on the concept of typical outage events and using ad-hoc approximations in different regimes, which were validated via MonteCarlo simulations but not via the analysis. Unlike [13][14], the present paper gives a rigorous analytical evaluation of the asymptotic outage probability (summarized in Theorems 1, 2 and Corollaries 1,2), from which the typical outage events of [13][14] follow as a consequence and do not require any ad-hoc assumptions or approximations.

Using the present approach, we demonstrate that there is a critical point below which the outage probability decays polynomially (i.e. slowly) but above which it decays exponentially (i.e. very fast), as a function of the threshold interference-tonoise ratio (INR), thus revealing a qualitative transition around this point. This provides a rigorous analytical foundation for the earlier empirical observations in the literature [9]. The analysis also reveals the outage-forming mechanism that is responsible for such behavior. The main analysis tools are the heavy tail distribution theory for the below-critical region and the saddle-point approximation theory for the above-critical one.

The rest of the paper is organized as follows. Section II introduces the system model. In section III, the rigorous asymptotic analysis of the outage probability is presented, and the non-asymptotic approximations are obtained based on it and validated via Monte-Carlo simulations. Section IV concludes the paper. The proofs are given in the Appendix.

\section{SYSTEM MODEL}

We consider a cognitive radio network which contains a primary user receiver and many secondary users' transmitters (nodes) on a plane. The PU is located at the origin. The SUs are randomly located according to a Poisson point process. The density of SUs is $\lambda$ [nodes $\left./ \mathrm{m}^{2}\right]$. Interference from the SU nodes outside the circle of a certain radius $R_{\max }$ is assumed to be negligible (alternatively, no SUs are located outside of this circle). The CR protocol is that all SUs which are inside of a forbidden (exclusion) region, i.e. the circle of the radius $R_{s}$ centered on the PU, cease their transmissions so that some protection to the PU is provided. The geometry of the CR network is illustrated in Fig. 1.

We assume that the desired signal, interferences and noise are independent of each other. The received power at the PU can be expressed as:

$$
P_{P U}=P_{d}+\sum_{i=1}^{N} I_{i}+P_{0}
$$

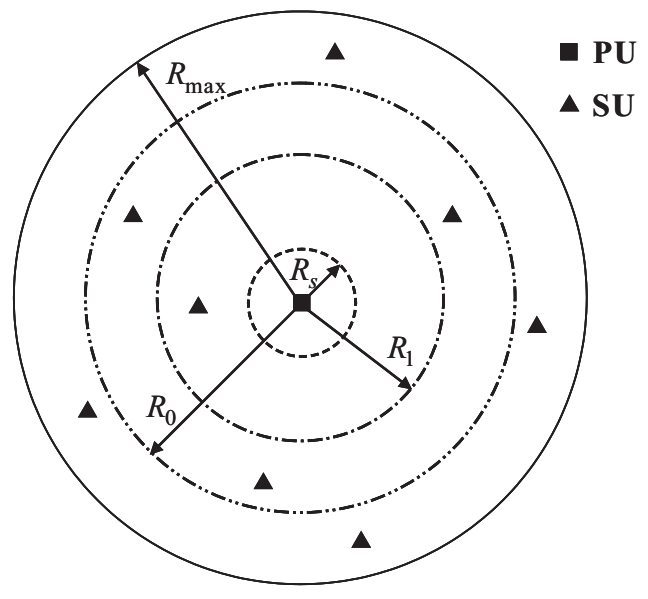

Fig. 1. The geometry of the CR network. Interference from the nodes outside the circle of the radius $R_{\max }$ is negligible. The interference coming from the nodes outside of the circle of radius $R_{0}$ is below the noise level. The average number of nodes in the disk of the radius $R_{1}$ is one. All SUs inside the circle of the radius $R_{s}$ centered on the $\mathrm{PU}$, the forbidden region, cease their transmissions.

where $P_{d}$ is the desired signal power; $I_{i}$ is the interference signal power coming from the $i^{\text {th }}$ node; $P_{0}$ is the noise power; $N$ is a Poisson random variable which denotes the number of nodes in the ring between circles of the radii $R_{s}$ and $R_{\max }$, i.e. the potential interference zone. The power at the receiver antenna input coming from a transmitter of power $P_{t}$ is given by the standard link budget equation, $P_{r}=P_{t} G_{t} G_{r} g$, where $G_{t}$ and $G_{r}$ are the transmitter and receiver antenna gains; $g$ is the propagation path gain. In this paper, we focus on the impact of the network geometry on the interference distribution (which is known to have a dominant contribution in regular networks [2]) and consider a non-fading scenario ${ }^{1}$ so that $g=a_{\nu} r^{-\nu}$ is the average path gain, where $\nu$ is the path loss exponent, $r$ is the distance between the transmitter and receiver, and $a_{\nu}$ is a constant independent of $r$; our method can also be extended to fading channels [15]. For simplicity, we assume the transmitter and receiver antennas are isotropic with unity gain, and all SUs transmit at the same constant power level $P_{s}$, so that $P_{r}=P_{s} g$. The $i^{t h} \mathrm{SU}$ generates the interference power $I_{i}=P_{s} a_{\nu} r_{i}^{-\nu}$ at the PU receiver, where $r_{i}$ is the distance between the $i^{t h} \mathrm{SU}$ and the PU. Without loss of generality, we normalize $P_{s} a_{\nu}=1$, so $I_{i}=r_{i}^{-\nu}$.

\section{Aggregate Interference Distribution And OUTAGE PROBABILITY}

When the signal to interference plus noise ratio (SINR) is less than a certain threshold $\eta$, there is significant performance degradation of a wireless link and it is considered to be in outage. This is equivalent, under the adopted channel model, to the aggregate interference power $I_{a g}$ exceeding the threshold $I_{t h}=P_{d} / \eta-P_{0}$, so that the outage probability is

\footnotetext{
${ }^{1}$ The results will also apply to fading scenarios and delay-insensitive applications, where the PU performance is determined by the average (over fading) powers [9].
} 


$$
P_{\text {out }}=\operatorname{Pr}\{\operatorname{SINR}<\eta\}=\operatorname{Pr}\left\{I_{a g}>I_{t h}\right\}
$$

Defining the INR as

$$
\gamma=\sum_{i=1}^{N} I_{i} / P_{0}
$$

Its threshold value is $D=I_{t h} / P_{0}$, so that the outage probability is:

$$
P_{\text {out }}=\operatorname{Pr}\{\gamma>D\}=1-F(D)
$$

where $F(D)$ is the CDF of the INR.

\section{A. Asymptotic Results}

In this section, we present our main contributions in Theorem 1 and 2, and Corollaries 1, 2, which are further extended to the non-asymptotic regime in the next section.

Since the CR protocol forces all SU nodes inside the forbidden region of the radius $R_{s}$ to cease their transmissions, the interference generated by a single node can not exceed $I_{\max }=R_{s}^{-\nu}$, i.e. the value coming from an active $\mathrm{SU}$ node at the closest possible distance, so that the maximum INR caused by a single node is $D_{\max }=I_{\max } / P_{0}$. Let us compare the CR network with the forbidden region of the radius $R_{s}$ and a corresponding conventional (non-CR) network, which is identical to the CR one except that there is no forbidden region (i.e. $R_{s}=0$ ). The relationship of their respective outage probabilities $P_{\text {out }}$ and $P_{c}$ in the asymptotic (lowoutage) regime is characterized as follows.

Theorem 1: Let $D=d \cdot D_{\max }$, where the normalized threshold INR $d$ is bounded away from unity, $d<1$, and $D \rightarrow \infty$. The CR outage probability is then asymptotically proportional to the conventional one:

$$
P_{\text {out }}=\left(1-d^{2 / \nu}\right) P_{c} \cdot(1+o(1))
$$

where $o(\cdot)$ is the small $o$ function ${ }^{2}$.

Proof: see the Appendix.

From Theorem 1 and the corresponding result for the conventional network in [5], it follows that the aggregate interference tail is dominated by the nearest node when $d<1$, which is formalized below.

Corollary 1: Let $P_{n i}=\operatorname{Pr}\left\{I_{[1]}>I_{t h}\right\}$ be the probability that the nearest node interference exceeds the threshold (thus causing an outage event). When $d<1$ and $D \rightarrow \infty$,

$$
\begin{aligned}
P_{\text {out }} & =P_{n i} \cdot(1+o(1)) \\
& =\left(1-d^{2 / \nu}\right) N_{0} D^{-2 / \nu} \cdot(1+o(1))
\end{aligned}
$$

where $N_{0}=\pi \lambda R_{0}^{2}$ is the average number of nodes in the disk of radius $R_{0}$.

Proof: see the Appendix.

For finite but large $D$ when $D<D_{\max }$, the results above can serve as accurate approximations (without $o(1)$ term), as

$$
{ }^{2} y=o(x) \text { if } \lim _{D \rightarrow \infty} y / x=0 .
$$

we demonstrate below in Section III-B. Note that (6) gives the tail behavior of $P_{\text {out }}$ with a guaranteed accuracy level as an explicit function of geometrical and system parameters.

While Theorem 1 holds for the $D<D_{\max }$ case, the opposite case of $D>D_{\max }$ is also of considerable practical interest for CR networks. In fact, while the outage probability decreases only polynomially with $D$ in the 1 st case (i.e. comparatively slowly), Corollary 2 below shows that it decreases exponentially in the latter case (i.e. very fast), thus revealing a remarkable qualitative transition around the $D=D_{\max }$ point.

To better understand why this happens, observe that $d<1$ in Theorem 1 implies $D<D_{\max }$, so that single-node interference can exceed the threshold and cause an outage event. Corollary 1 further demonstrates that this is a typical outage event. On the other hand, when $D>D_{\max }$, the single-node interference can not exceed the threshold so that a combined effect of several nodes is required. Let $n=\left\lceil D / D_{\max }\right\rceil$, where $\lceil x\rceil=\min \{n \in \mathbb{Z} \mid n \geq x\}$ is a ceiling function. The outage occurs when the combination of at least $n$ nodes' interference exceeds the threshold. When $D_{\max }<\infty$ and $D \rightarrow \infty$, we have $n \rightarrow \infty$, so that some form of the central limit theorem (CLT) should apply, which results in the Gaussian approximation being a main candidate. As is well-known, this applies around the mean of the distribution but quickly deteriorates as one moves to the distribution tail, i.e. the region we are interested in (that is the low-outage region), when $n$ is not sufficiently large. To overcome this problem, we use the saddle-point theory (also known as the tilted Edgeworth expansion), which suggest tilting the original distribution in a way that the point of interest is always around the mean (akin to the importance sampling technique) [12]. An immediate effect is that the relative rather than absolute error becomes small, which has a dramatic positive consequence for the distribution tail.

A summary of the saddle-point approximation theory is given in Lemma 1 in the Appendix. Based on it, we have the following result.

Theorem 2: When $D_{\max }<\infty$ and $D \rightarrow \infty$, which implies $D>D_{\max }$, the CR outage probability can be expressed as:

$$
P_{\text {out }}=Q\left(z+z^{-1} \ln \sqrt{z^{-2} \hat{\theta}^{2} d_{1} N_{s}^{-\nu / 2}}\right)(1+o(1))
$$

where $Q(x)=1 / \sqrt{2 \pi} \int_{x}^{\infty} \exp \left(-u^{2} / 2\right) d u$ is the standard Q-function, $z=\left(2 d_{1}\left(\hat{\theta}-N_{s}^{\nu / 2}\right)-2 N_{s}\right)^{1 / 2}, d_{1}=D / D_{1}$, $D_{1}=1 / R_{1}^{\nu} P_{0}=(\pi \lambda)^{\nu / 2} / P_{0}$, and $R_{1}$ is the radius of the disk with on average one node in it (so that $D_{1}$ is the INR from the boundary of that disk ${ }^{3}$ ); $N_{s}=\pi \lambda R_{s}^{2}$ is the average number of nodes in the forbidden region of the radius $R_{s}$, and

$$
\hat{\theta}=N_{s}^{\nu / 2}(\ln w+\ln \ln w)+o(\ln \ln w)
$$

is the saddle-point, where $w=\nu d_{1} N_{s}^{\nu / 2-1} / 2(\nu>2)$.

\footnotetext{
${ }^{3}$ It is also a critical value which separates the high and low outage probability regions in non-CR networks.
} 
Proof: see the Appendix.

In the next section, we show that (7) can be used as an accurate approximation (without $o(1)$ term) for finite but large $D$ when $D>D_{\max }$, so that the saddle-point approach reduces the approximation error significantly compared to the Gaussian or Edgeworth approximations. Further analysis shows that, unlike the outage probability in (6) which decays polynomially in $D$, that in (7) decays exponentially, i.e. much faster [15].

Corollary 2: When $D_{\max }<\infty$ and $D \rightarrow \infty$, the outage probability in (7) scales as

$$
P_{\text {out }}=\exp \left\{-N_{s}^{\nu / 2} d_{1} \ln d_{1}(1+o(1))\right\}
$$

where $d_{1}=D / D_{\max }$. This qualitative transition around the $D=D_{\max }$ point is in agreement with the earlier empirical observations in [9].

\section{B. Non-asymptotic outage probability}

The asymptotic results above can be used as approximations (without $o(1)$ terms) for finite but large $D$ under certain conditions, which we summarize below based on the concept of typical outage events by considering 3 typical cases.

- Case 1: $D_{1} \ll D<D_{\max }$.

From Theorem 1 and Corollary 1 , for finite but large $D$ and $D<D_{\max }, P_{\text {out }} \approx P_{n i}$. The key question is: At what value is $D$ sufficiently large so that the approximation is accurate? From [5], the nearest interferer approximation is accurate in the non-CR networks when $D \gg D_{1}$, where $D_{1}=(\pi \lambda)^{\nu / 2} / P_{0}$ is a critical value which separates the high and low outage probability regions, and is the INR coming from the boundary of the disk (centered on the PU) with on average one node in it. As Theorem 1 links $P_{\text {out }}$ and $P_{c}$, the corresponding condition also applies to the former, so that large $D$ means $D_{\max }>D \gg D_{1}$, the aggregate interference is dominated by the nearest node and $P_{\text {out }} \approx P_{n i}$. This is possible when $D_{1} \ll D_{\max }$, which implies $R_{s} \ll R_{1}$, i.e. a small forbidden region.

Fig. 2 shows the outage probability in this case. When $D_{1} \ll D<D_{\max }$, it is well approximated by the nearest node $P_{n i}$. On the other hand, the nearest node interference cannot exceed $D_{\max }$, so the above approximation breaks down when $D>D_{\max }$. These observation have been validated via extensive Monte-Carlo simulations for a wide range of system parameters, of which Fig. 2 is only a sample.

- Case 2: $R_{s} \ll R_{1}$ and $D>D_{\max }$, or $R_{s}<R_{1}$ but not $R_{s} \ll R_{1}$.

When $R_{s} \ll R_{1}$ and $D>D_{\max }$, or $R_{s}<R_{1}$ but not $R_{s} \ll R_{1}$, for practically-important outage range (say $P_{\text {out }}>10^{-10}$ ), the number $n$ of nodes contributing to a typical outage event is not sufficiently large and Gaussian or Edgeworth approximations are not accurate, as Fig. 2 shows. On the other hand, as explained above, the saddle-point method tilts the original distribution and makes the relative error small so that its accuracy is much better at the tail.

Fig. 2 shows the saddle-point approximation when $R_{s} \ll$ $R_{1}$. When $D>D_{\max }$, its accuracy is remarkably good. On the

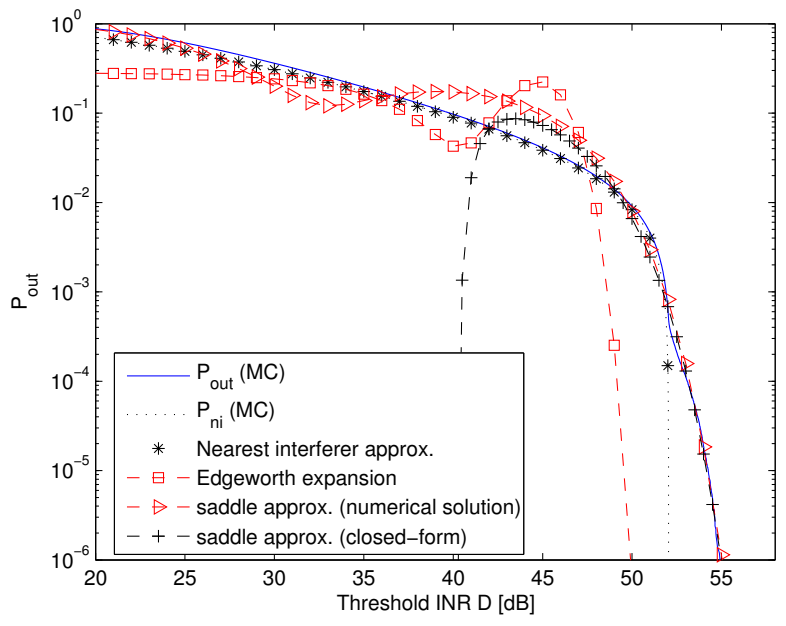

Fig. 2. Outage probability when $R_{s} \ll R_{1}$. The simulation parameters are $\nu=4, R_{s}=10 \mathrm{~m}, R_{1}=56 \mathrm{~m}, R_{0}=200 \mathrm{~m}, R_{\max }=10^{3} \mathrm{~m}$, $\lambda=10^{-4}\left[\right.$ nodes $\left./ m^{2}\right], D_{1}=22 \mathrm{~dB}, D_{\max }=52 \mathrm{~dB}$. MC denotes MonteCarlo simulations.

other hand, when $D<D_{\max }$, the saddle-point approximation is not accurate since the aggregate interference is dominated by the nearest node and the CLT-type argument is not expected to work well in this case. Extensive Monte-Carlo simulations confirm that arguments hold for a wide range of system parameters.

- Case 3: $R_{s}>R_{1}$.

When $R_{s}>R_{1}$, i.e. a large forbidden region, $n$ is large in the low outage range, i.e. a typical outage event is when the combination of interference from many nodes exceeds the threshold, and the aggregate interference is closely approximated by a Gaussian random variable, $\sum_{i=1}^{N} I_{i} \sim \mathcal{N}$ [9][14]. As Fig. 3 demonstrates, the Gaussian and Edgeworth approximations are accurate with $1-2 \mathrm{~dB}$ gap at the tail, and the saddle-point approximation is the most accurate one with $0.1 \mathrm{~dB}$ gap at the tail. The closed-form expression of the saddle-point approximation is less accurate than the numerical one, since the former was based on the asymptotic solution.

\section{CONCLUSION}

This paper provided the accurate, closed-form outage provability expressions for CR networks, which are based on the asymptotic analysis and the saddle-point theory and are an important evaluation tool in the deployment of the future $\mathrm{CR}$ networks. The asymptotic analysis revealed the qualitative transition in the outage probability behavior around the critical point, when the decay changes from polynomial to exponential, thus providing a rigorous analytical foundation for the earlier empirical observations in the literature. All results and conclusions are validated via extensive MonteCarlo simulations.

\section{APPENDIX}

\section{Proof of Theorem 1:}




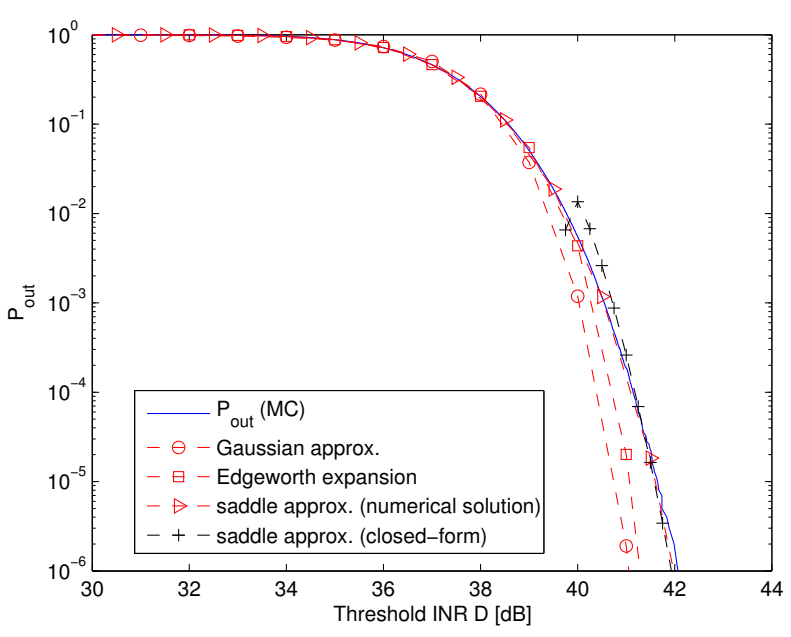

Fig. 3. Outage probability when $R_{s}>R_{1}$. The simulation parameters are $\nu=4, R_{s}=32 \mathrm{~m}, R_{1}=18 \mathrm{~m}, R_{0}=200 \mathrm{~m}, R_{\max }=10^{3} \mathrm{~m}$, $\lambda=10^{-3}\left[\right.$ nodes $\left./ m^{2}\right], D_{1}=42 \mathrm{~dB}, D_{\max }=32 \mathrm{~dB}$. MC denotes MonteCarlo simulations.

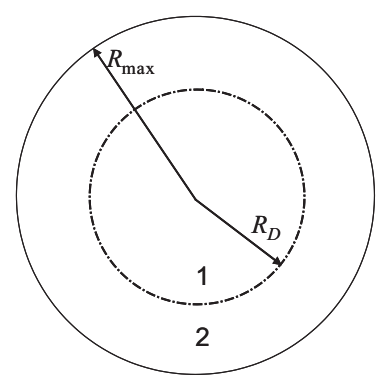

(a) non-CR wireless network

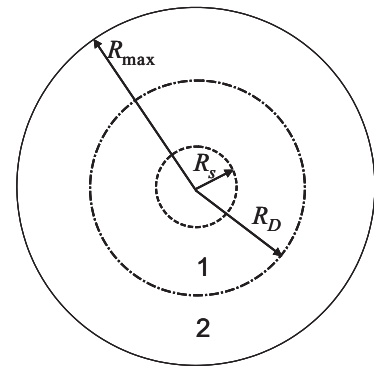

(b) Cognitive radio network
Fig. 4. Geometry of non-CR and CR wireless networks

Let $n_{D}$ be the number of nodes in disk 1 of the radius $R_{D}$ in Fig. 4.a, and $n_{1}$ be the number of nodes in ring 1 which is between the circles with radii $R_{s}$ and $R_{D}$ in Fig. 4.b. Let ring 2 be the ring between the circles with radii $R_{D}$ and $R_{\max }$ in Fig. 4. Define the following probabilities: $P_{1}=\operatorname{Pr}\left\{n_{D} \geq 1\right\}$, $P_{2}=\operatorname{Pr}\left\{\sum_{i \subseteq \text { ring } 2} I_{i}>D P_{0}\right\}, P_{3}=\operatorname{Pr}\left\{n_{1} \geq 1\right\}$. The non$\mathrm{CR}$ probability can be now expressed as:

$$
P_{c}=P_{1}+\left(1-P_{1}\right) P_{2}
$$

From the Poisson point process, $P_{1}=1-\exp \left(-\lambda \pi R_{D}^{2}\right)$, and from Theorem 1 in [5], as $D \rightarrow \infty$,

$$
\left(1-P_{1}\right) P_{2} / P_{1}=o(1)
$$

The CR outage probability can be expressed as:

$$
P_{\text {out }}=P_{3}+\left(1-P_{3}\right) P_{2}
$$

Since $D=d \cdot D_{\max }$, one obtains $P_{3}=1-\exp \left[-\lambda \pi R_{D}^{2}(1-\right.$ $\left.d^{2 / \nu}\right)$ ]. When $d<1$ and $D \rightarrow \infty$, the limit of the ratio of the $\mathrm{CR}$ and non-CR outage probabilities can be evaluated as follows:

$$
\begin{aligned}
\lim _{D \rightarrow \infty} \frac{P_{\text {out }}}{P_{c}} & =\lim _{D \rightarrow \infty}\left(\frac{P_{3}}{P_{1}}+\frac{\left(1-P_{3}\right) P_{2}\left(1-P_{1}\right)}{P_{1}\left(1-P_{1}\right)}\right) \\
& \times\left(1+\frac{\left(1-P_{1}\right) P_{2}}{P_{1}}\right)^{-1}=\left(1-d^{2 / \nu}\right)
\end{aligned}
$$

from which (5) follows.

\section{Proof of Corollary 1:}

From [[5] Theorem 1], when $D \rightarrow \infty$,

$$
P_{c}=N_{0} D^{-2 / \nu} \cdot(1+o(1))
$$

where $N_{0}=\pi \lambda R_{0}^{2}$. When $d<1$ and $D \rightarrow \infty$, one obtains from (5) and (12),

$$
P_{\text {out }}=N_{0}\left(D^{-2 / \nu}-D_{\max }^{-2 / \nu}\right) \cdot(1+o(1))
$$

where $D_{\max }=D / d$. On the other hand, using the Poisson point process probabilities when $d<1$,

$$
P_{n i}=N_{0}\left(D^{-2 / \nu}-D_{\max }^{-2 / \nu}\right)
$$

Comparing (13) and (14), when $d>1$ and $D \rightarrow \infty, P_{\text {out }}=$ $P_{n i} \cdot(1+o(1))$.

\section{Proof of Theorem 2:}

The following lemma summarizes the main saddle-point approximation results and is instrumental for the further analysis.

Lemma 1: Let $Y_{1}, \cdots, Y_{n}$ be independent and identically distributed (i.i.d) random variables with the Moment Generation Function (MGF) $M(\theta)$ with $\sup \{\theta: M(\theta)<\infty\}=\tau_{2}$, and $S_{n}=\sum_{i=1}^{n} Y_{i}$, where $n$ is a Poisson random variable with mean $v$ with the MGF

$$
M_{c}(\theta)=E\left[\exp \left(\theta S_{n}\right)\right]=\exp \{v[M(\theta)-1]\}
$$

where the subscript $c$ stands for compound.

When $M(\theta)$ is finite for $\theta<\tau_{2}$ with $\tau_{2}>0$, and $M(\theta) \rightarrow$ $\infty$ for $\theta \rightarrow \tau_{2}$, then, the probability of that $S_{n}$ is greater than or equal to threshold $s$ is

$$
\operatorname{Pr}\left(S_{n} \geq s\right)=Q\left(R_{L}\right)\left\{1+O\left[\rho_{c 4}(\hat{\theta})\right]\right\}
$$

where $\hat{\theta}$ is the saddle point which is the solution of $v M^{\prime}(\theta)=$ $s$;

$$
R_{L}=R+R^{-1} \ln (U / R)
$$

and

$$
\rho_{c k}(\hat{\theta})=\frac{\mathrm{K}_{c}^{(k)}(\hat{\theta})}{\left[\sigma_{c}(\hat{\theta})\right]^{k}}=\frac{v M^{(k)}(\hat{\theta})}{\left[v M^{\prime \prime}(\hat{\theta})\right]^{k / 2}} .
$$

In $R_{L}$,

$$
R=\operatorname{sgn}(\hat{\theta})(2 \hat{\theta} s-2 v(M(\hat{\theta})-1))^{1 / 2}
$$

and

$$
U=\hat{\theta} \sigma_{c}(\hat{\theta})=\hat{\theta} \sqrt{v M^{\prime \prime}(\hat{\theta})}
$$

where $\operatorname{sgn}(x)$ is a sign function, that is, $\operatorname{sgn}(x)=1$ for $x \geq 0$ and $\operatorname{sgn}(x)=-1$ for $x<0$. 
The proof is given in [[12], Ch.7].

One important property of Poisson point process is that the number of nodes in the region $S$ is independent with that in any other disjoint region. Based on that, the distances between a random picked node and the origin on a plane are independent with each other, so that the interference powers coming from a single node without ordering are i.i.d random variables in non-fading channels.

The pdf of the interference power $I$ coming from a single node (without ordering) in non-fading channels is:

$$
f(I)=\left\{\begin{array}{cc}
\frac{2}{\nu} \frac{I^{-1-2 / \nu}}{R_{\max }^{2}-R_{s}^{2}} & R_{\max }^{-\nu} \leq I \leq R_{s}^{-\nu} \\
0 & \text { otherwise }
\end{array}\right.
$$

Let $x_{1}, \cdots, x_{N}$ be the INRs coming from a single node without ordering after normalizing by $D_{1}$, so that the pdf of $x=I /\left(P_{o} D_{1}\right)$ is

$$
f(x)=\left\{\begin{array}{cc}
\frac{2}{\nu} \frac{x^{-1-2 / \nu}}{N_{r}} & N_{\max }^{-\nu / 2} \leq x \leq N_{s}^{-\nu / 2} \\
0 & \text { otherwise }
\end{array}\right.
$$

where $N_{r}=\pi \lambda\left(R_{\max }^{2}-R_{s}^{2}\right)$ is the average number of nodes in the ring between the circles with radii $R_{\max }$ and $R_{s} ; N_{\max }=$ $\pi \lambda R_{\max }^{2}$ is the average number of nodes in the disk of the radius $R_{\text {max }} ; N_{s}=\pi \lambda R_{s}^{2}$ is the average number of nodes in the disk of the radius $R_{s}$.

The MGF of $x$ can be expressed as:

$$
M_{x}(\theta)=\frac{2}{\nu N_{r}} \int_{N_{\max }^{-\nu / 2}}^{N_{s}^{-\nu / 2}} \exp (\theta x) x^{-1-2 / \nu} d x
$$

When $D_{\max }<\infty$, we have $0<N_{s}^{-\nu / 2}<\infty$. Since $0<$ $N_{\max }^{-\nu / 2}<\infty, 0<N_{s}^{-\nu / 2}<\infty$, and $0<\exp (\theta x) x^{-1-2 / \nu}<$ $\infty$ with $N_{\max }^{-\nu / 2}<x<N_{s}^{-\nu / 2}$ and $\theta<\infty$, we have $0<$ $M_{x}(\theta)<\infty$, and

$$
\sup \left\{\theta: M_{x}(\theta)<\infty\right\}=\infty
$$

It satisfies the conditions of Lemma 1, so the saddle-point approximation in Lemma 1 can be used.

Firstly, we find the asymptotic solution of the saddle point $\hat{\theta}$ by solving $N_{r} M_{x}^{\prime}(\theta)=d_{1}$, where $d_{1}=D / D_{1}$. After changing variables, the equation is $d_{1}=p \int_{a}^{b} \exp (\theta x) x^{-p} d x$, where $p=2 / \nu<1,(\nu>2) ; a=N_{\max }^{-\nu / 2}$ and $b=N_{s}^{-\nu / 2}$. When $d_{1} \rightarrow \infty$, we have $\theta \rightarrow \infty$. Using integration by parts, the asymptotic solution is:

$$
d_{1}=\frac{p \exp (\theta b)}{\theta b^{p}}[1+o(1)]
$$

The asymptotic solution of $\hat{\theta}$ (keeping two terms) is:

$$
\begin{aligned}
\hat{\theta} & =\frac{1}{b} \ln \left(\frac{d_{1} b^{p-1}}{p}\right)+\frac{1}{b} \ln \ln \left(\frac{d_{1} b^{p-1}}{p}\right)(1+o(1)) \\
& =N_{s}^{\nu / 2}(\ln w+\ln \ln w)+o(\ln \ln w)
\end{aligned}
$$

where $w=\nu d_{1} N_{s}^{\nu / 2-1} / 2$.
Secondly, we calculate $R$ and $U . R=$ $\operatorname{sgn}(\hat{\theta})\left\{2 d_{1}\left(\hat{\theta}-b^{-1}\right)-2 N_{s}\right\}^{1 / 2}$ and $U=\hat{\theta} \sqrt{d_{1} b}$. Let $z=\sqrt{2 d_{1}\left(\hat{\theta}-N_{s}^{\nu / 2}\right)-2 N_{s}}$, so that

$$
R_{L}=z+z^{-1} \ln \left(z^{-1} \hat{\theta} \sqrt{d_{1} N_{s}^{-\nu / 2}}\right)
$$

Thirdly, the asymptotic solution of $\rho_{c 4}(\theta)$ is

$$
\begin{aligned}
\rho_{c 4}(\theta) & =\frac{\nu}{2} \frac{\int_{N_{\max }^{-\nu / 2}}^{N_{-\nu / 2}^{-\nu}} \exp (\theta x) x^{3-2 / \nu} d x}{\left[\int_{N_{\max }^{-\nu / 2}}^{N_{s}^{-\nu / 2}} \exp (\theta x) x^{1-2 / \nu} d x\right]^{2}} \\
& =\frac{\nu b}{2} \frac{\theta b^{2 / \nu}}{\exp (\theta b)}[1+o(1)]
\end{aligned}
$$

From (21),

$$
\rho_{c 4}(\hat{\theta})=\frac{b}{d_{1}}[1+o(1)]
$$

and using (24) in (16), one finally obtains

$$
P_{\text {out }}=Q\left(R_{L}\right)[1+o(1)]
$$

\section{REFERENCES}

[1] Special Issue: "Cognitive Radio Part 2: Fundamental Issues", Proceedings of the IEEE, vol. 97, no.5, May 2009.

[2] M. Haenggi and R.K. Ganti, Interference in Large Wireless Networks, Foundations and Trends in Networking, v. 3, N. 2, pp. 127-248, 2009.

[3] E.S. Sousa and J.A. Silvester, "Optimum transmission ranges in a directsequence spread-spectrum multihop packet radio network," IEEE Journal on Selected Areas in Communications, vol.8, no.5, pp.762-771, Jun 1990.

[4] J. Ilow and D. Hatzinakos, "Analytic alpha-stable noise modeling in a Poisson field of interferers or scatterers," IEEE Transactions on Signal Processing, vol.46, no.6, pp.1601-1611, Jun 1998.

[5] V. Mordachev and S. Loyka, "On node density - outage probability tradeoff in wireless networks," IEEE Journal on Selected Areas in Communications, vol.27, no.7, pp.1120-1131, Sept. 2009.

[6] O.B.S. Ali, C. Cardinal and F. Gagnon, "On the Performance of Interference Cancellation in Wireless Ad Hoc Networks", IEEE Trans. Comm. v. 58, N. 2, pp. 433-437, Feb. 2010.

[7] M. Aljuaid and H. Yanikomeroglu, "Investigating the Gaussian Convergence of the Distribution of the Aggregate Interference Power in Large Wireless Networks", IEEE Trans. Vechicular Tech., v. 59, N. 9, pp. 44184424, Nov. 2010.

[8] A. Ghasemi and E.S. Sousa, "Interference Aggregation in SpectrumSensing Cognitive Wireless Networks," IEEE Journal of Selected Topics in Signal Processing, vol.2, no.1, pp.41-56, Feb. 2008.

[9] X. Hong, C.-X. Wang and J. Thompson, "Interference Modeling of Cognitive Radio Networks", IEEE VTC Spring 2008, Singapore, May 2008.

[10] A. Rabbachin, T.Q.S. Quek, H. Shin and M.Z. Win, "Cognitive Network Interference ," IEEE Journal on Selected Areas in Communications, vol.29, no.2, pp.480 - 493, Jan. 2011.

[11] A.J. Ganesh and G.L. Torrisi, "Large Deviations of the Interference in a Wireless Communication Model," IEEE Transactions on Information Theory, vol.54, no.8, pp.3505 -3517, Aug. 2008.

[12] J.L. Jensen, Saddlepoint Approximation. Oxford University Press, Oxford, 1995.

[13] Y. Wen, S. Loyka and A. Yongacoglu, "The Impact of Fading on the Outage Probability in Cognitive Radio Networks," IEEE VTC 2010-Fall, Sept. 2010

[14] Y. Wen, S. Loyka and A. Yongacoglu, On Distribution of Aggregate Interference in Cognitive Radio Networks, 25th Biennial Symposium on Communications (QBSC-10), May 2010.

[15] Y. Wen, Outage Probability and Agregate Interference in Cognitive Radio Networks, PhD Thesis, University of Ottawa, 2012. 